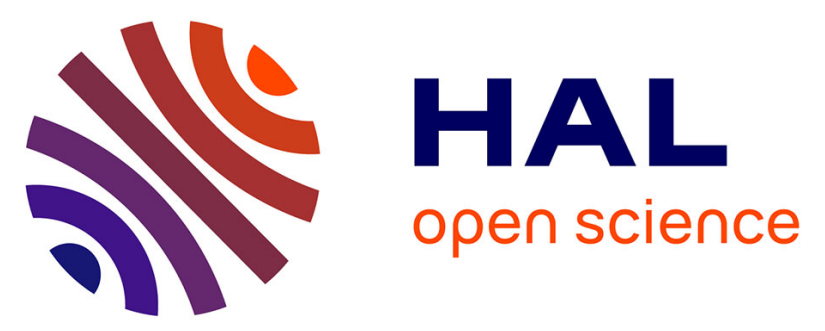

\title{
Antidepressant short-term and long-term brain effects during self-referential processing in major depression
}

Pauline Delaveau, Maritza Jabourian, Cédric Lemogne, Najib Allaili, Walid

Choucha, Nathalie Girault, Stéphane Lehericy, Judith Laredo, Philippe

Fossati

\section{To cite this version:}

Pauline Delaveau, Maritza Jabourian, Cédric Lemogne, Najib Allaïli, Walid Choucha, et al.. Antidepressant short-term and long-term brain effects during self-referential processing in major depression. Psychiatry Research: Neuroimaging, 2015, 247, pp.17-24. 10.1016/j.pscychresns.2015.11.007 . hal01243633

\section{HAL Id: hal-01243633 \\ https://hal.sorbonne-universite.fr/hal-01243633}

Submitted on 15 Dec 2015

HAL is a multi-disciplinary open access archive for the deposit and dissemination of scientific research documents, whether they are published or not. The documents may come from teaching and research institutions in France or abroad, or from public or private research centers.
L'archive ouverte pluridisciplinaire HAL, est destinée au dépôt et à la diffusion de documents scientifiques de niveau recherche, publiés ou non, émanant des établissements d'enseignement et de recherche français ou étrangers, des laboratoires publics ou privés. 


\section{Antidepressant short-term and long-term brain effects during self-referential processing in major depression}

Pauline Delaveau $^{\mathrm{a},{ }^{*}}$, Maritza Jabourian ${ }^{\mathrm{d}}$, Cédric Lemogne ${ }^{\mathrm{e}, \mathrm{f}, \mathrm{g}}$, Najib Allaïli ${ }^{\mathrm{a}, \mathrm{h}}$, Walid Choucha ${ }^{\mathrm{b}}$, Nathalie Girault ${ }^{\mathrm{b}}$, Stéphane Lehericy ${ }^{\mathrm{a}, \mathrm{h}, \mathrm{i}}$, Judith Laredo ${ }^{\mathrm{d}}$, Philippe Fossati ${ }^{\mathrm{a}, \mathrm{b}, \mathrm{c}}$

annserm U 1127, CNRS UMR 7225, Sorbonne Universités, UPMC Univ Paris 06, UMR S 1127, Institut du

Cerveau et de la Moelle, ICM, Social and Affective Neuroscience (SAN) Laboratory, Paris, France

${ }^{\mathrm{b}}$ AP-HP, Department of Psychiatry, Pitié-Salpêtrière Hospital, Paris, France

${ }^{c}$ Institut de Recherche Translationnelle en Neurosciences ICM-A-IHU, Paris, France

${ }^{\mathrm{d} I n s t i t u t ~ d e ~ R e c h e r c h e s ~ I n t e r n a t i o n a l e s ~ S e r v i e r, ~ S u r e s n e s, ~ F r a n c e ~}$

${ }^{e}$ Université Paris Descartes, Sorbonne Paris Cité, Faculté de Médecine, Paris, France

${ }^{\mathrm{f}}$ AP-HP, Hôpitaux Universitaires Paris Ouest, Service de Psychiatrie de l'adulte et du sujet âgé, Paris, France

${ }^{\mathrm{g}}$ Inserm U894, Centre Psychiatrie et Neurosciences, Paris, France

${ }^{\mathrm{h}}$ Institut du Cerveau et de la Moelle épinière - ICM, Centre de NeuroImagerie de Recherche - CENIR, Paris, France

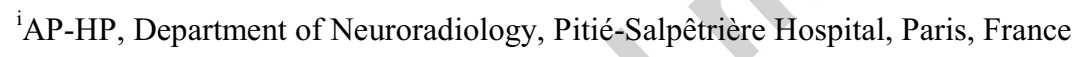

*Corresponding author: Tel.: +33 (0)1 421616 55; Fax: +33 (0)1 537907 70; Email:

pauline.delaveau@yahoo.fr

\section{Abstract}

Acute depression is associated with impaired self-referential processing. Antidepressant effects on the neural bases of self-referential processing in depression are unknown. This study aimed to assess short- and long-term effects of agomelatine on these neural bases in depressed patients and the association between pre-treatment brain activation and remission of depression 6 months later. We conducted a randomized double-blind, placebo-controlled, functional magnetic resonance imaging (fMRI) study during an emotional self-referential task, including three scanning sessions (baseline, after 1 week, and after 7 weeks). Twenty- 
five depressed outpatients were included, all treated with agomelatine or placebo for 1 week. Then, all patients received agomelatine for 24 weeks. Fourteen matched healthy volunteers (HV) who received placebo for 1 week were also included. After 7 days, only depressed patients receiving agomelatine significantly deactivated the ventrolateral prefrontal cortex during self-referential processing, as observed in $\mathrm{HV}$ at baseline. After 7 weeks, depressed patients significantly increased the activation of the ventral anterior cingulate cortex. Finally dorsomedial prefrontal cortex and precuneus activations at baseline significantly separated remitters from non-remitters at 24 weeks. In depressed patients, agomelatine had short- and long-term effects on brain structures involved in anhedonia and emotional regulation during self-referential processing. Activation of the dorsomedial prefrontal cortex and precuneus could be informative in the development of biomarker-based treatment of major depression.

[Trials registration: Registered at controlled-trials.com (ISRCTN53505070)]

Keywords: Major depressive disorder, Functional magnetic resonance imaging (fMRI), Antidepressant, Self-referential processing, Short-term, Long-term

\section{Introduction}

Major depressive disorder (MDD) is associated with several biases in information processing. Depressed patients prioritize the processing of negative emotional information and show increased attention to the self, namely self-focus (Mor and Winquist, 2002). Increased selffocus in depressed patients involves the cortical midline structures, including the medial prefrontal cortex (MPFC) and the posterior cingulate cortex (PCC) (Lemogne et al., 2009; Yoshimura et al., 2010; Lemogne et al., 2012; Nejad et al., 2013), as well as the dorsolateral prefrontal cortex (DLPFC; Lemogne et al., 2009). 
There is growing interest in the effects of antidepressant drugs on such biases as they play a key role in the initiation and maintenance of depressive symptoms (Harmer et al., 2003, 2004, 2009; Pringle et al., 2011). On one hand, one pilot study showed that long-term administration of antidepressants in depressed patients modulates the activity of the DLPFC during selfprocessing (Lemogne et al., 2010). On the other hand, although some studies with short-term administration of selective serotonin reuptake inhibitors in healthy subjects showed a modulation of midline cortical regions (Mattews et al., 2010; Di Simplicio et al., 2012), shortand long-term neural correlates of antidepressant effects on self-focus in depressed patients are still little known.

The main goal of the present functional magnetic resonance imaging (fMRI) study was to assess both the short- and long-term effects of an antidepressant on the neural correlates of increased self-focus in depressed patients. Here, we used agomelatine, a potent agonist of $\mathrm{MT}_{1}-\mathrm{MT}_{2}$ melatoninergic receptors and an antagonist of the serotoninergic 5-HT2 $\mathrm{C}$ receptor (de Bodinat et al., 2010) with established antidepressant efficacy (Taylor et al., 2014).

Since increased self-focus can be defined as a tendency to engage in self-referential processing, we assessed depressed patients making self-referential judgments on emotional pictures during the following three fMRI scanning sessions: before treatment when patients were acutely depressed, after 1 week of treatment with agomelatine or placebo, and after 6 to 7 weeks of agomelatine.

We predicted that treatment with agomelatine, compared with placebo, would induce in depressed patients early changes in the MPFC and lateral prefrontal cortex (PFC) even in the absence of significant changes in mood ratings and depressive symptoms. Second, we hypothesized that 6-7 weeks of treatment with agomelatine would induce changes in the activity of the medial and lateral PFC, concomitantly with a clinical improvement of 
depression. Finally, we tested if the activity of brain regions associated with self-referential processing at baseline would be associated with clinical remission 6 months later.

\section{Methods}

\subsection{Subjects}

Thirty female outpatients meeting DSM-IV criteria for MDD with the Mini International Neuropsychiatric Interview (MINI, Sheehan et al., 1998) were recruited between October 2008 and June 2011 by psychiatrists ( 7 centres located in Paris area, France).

Current depressive episode had to be unipolar subtype, longer than 4 weeks, of moderate or severe intensity with total scores $\geq 22$ on the 17 -item Hamilton Rating Scale for Depression (HAM-D; Hamilton, 1960) and Clinical Global Impression (CGI) severity scores $\geq 4$ (Guy, 1976). All patients had to be free of psychotropic medication for a minimum of 2 weeks (washout period defined according to the medication) before inclusion. Patients with comorbid conditions were excluded (see Supplementary Material 1 for details).

Two subgroups of 15 patients were determined by the blinded allocation of the treatment for the first week: agomelatine (AGO) or placebo (PBO) (see Section 2.2). Two patients of the AGO group and three patients of the PBO group were excluded (one because of excessive head movements in the MRI scanner and four owing to positive drug screening) leading to a final sample of 13 patients in the AGO group and 12 patients in the PBO group. These MDD patients were compared with the $14 \mathrm{HV}$. All groups were matched for age and gender (see supplementary Fig. 1 for CONSORT flow diagram).

Table 1 summarizes demographic and clinical characteristics of the MDD and HV groups. This study was conducted in accordance with the principles of the Declaration of Helsinki. Approval was obtained from the French ethics committee for Biomedical Research of the Pitié-Salpêtrière Hospital (Paris). Each participant gave his/her informed written consent 
before entering the study, and healthy volunteers were registered on the French National File.

\subsection{Drug treatment and study design}

The study was randomized, double-blind, multicenter and placebo-controlled during the first week, with two parallel groups of MDD patients (AGO vs. PBO). Patients took one tablet of AGO $25 \mathrm{mg}$ or placebo, orally per day at around 8 p.m., for 1 week (except for one patient in the AGO group who missed 1 day of treatment) in a double-blind protocol followed by an open-label period with AGO until 24 weeks (W24). At W7, patients had thus received 6 or 7 weeks of treatment with AGO, with two possible sequences of treatment (PBO/AGO, AGO/AGO). All HV took one tablet of placebo per day orally at around 8 p.m., during 1 week in a single-blind protocol (see Supplementary Material 2 for randomization).

Each patient was scanned on three occasions, before beginning treatment (W0), after 1 week (W1) and 7 weeks of treatment (W7). Among the 25 MDD patients, all were included for analyses regarding the short-term AGO effects after 1 week (part I), but six patients had missing fMRI data at W7 (see CONSORT flow diagram, supplementary Fig. 1), leading to a final sample of 19 MDD patients for the long-term (W0-W7) AGO effects (part II). The HV were scanned on two occasions, at W0 and after 1 week of PBO (supplementary Fig. 2).

\subsection{Clinical assessment}

Depression severity was assessed with the 17-item HAM-D scale (Hamilton, 1960) and CGI severity and clinical improvement subscores (Guy, 1976) before each fMRI scan (W0, W1, W7) and after 24 weeks of treatments (W24; three missing HAM-D assessments from the final sample of 19 patients leading to a sub-sample of 16 patients for analyses regarding remission prediction, part III). 


\subsection{Experimental design}

A total of 432 black and white pictures were taken from either the International Affective Picture System (IAPS, Lang et al., 2005) or the Empathy Picture System (EPS, Geday et al., 2003) (Supplementary Material 3).

The task comprised three judgment conditions: self, general and a control (Lemogne et al., 2011). In both self and general conditions, the subjects were presented with an equal number of positive and negative pictures. In the control condition, subjects were presented with neutral pictures. In the self condition, the subject judged if the picture was self-related or not. In the general condition, the subject judged if the picture was positive or negative. In the control condition, the subject had to state if the picture represented an outdoor or indoor scene (supplementary Fig. 3).

\section{5. fMRI protocol}

All stimuli were back-projected onto a semi-transparent screen located at the side of the head that the subjects viewed through a 45-degree mirror above their eyes.

Before fMRI scanning, subjects performed a practice run similar to functional runs outside the scanner. The fMRI design consisted of three functional runs of 36 images each. Each run comprised three blocks, each block associated with only one condition ("self", "general", or "control") in a pseudo-random counterbalanced order across the runs. For each condition there were six positive images, six negative images for self and general conditions, or six indoor and six outdoor neutral pictures for the control condition. Each trial consisted of a picture displayed for $2.565 \mathrm{~s}$, followed by a fixation cros-shair for $3.5 \mathrm{~s}$. The duration of each trial (i.e., $6.065 \mathrm{~s}$ ) was computed to optimize the hemodynamic response sampling over the course of each block, taking into account the echo-planar imaging (EPI) sequence repetition time. To introduce jitter into the fMRI time series, each block contained six null events, 
consisting of a fixation cross-hair for $6.065 \mathrm{~s}$. Pictures and null events were pseudo-randomly intermixed. The lists were drawn up randomly for each subject.

The start of each list was preceded by an instruction cue specifying for $5.330 \mathrm{~s}$ the judgment condition (self, general or control) followed by presentation of a fixation cross-hair for $3.5 \mathrm{~s}$ to allow a decrease in the hemodynamic response (supplementary Fig. 3).

\section{6. fMRI data acquisition}

All data acquisition was performed in a 3 T TRIO 32-channel TIM scanner (Siemens Medical Solutions, Erlangen, Germany), in the Centre for NeuroImaging Research of the Salpêtrière Hospital, with a 12-channel head coil. The fMRI scans were obtained using a T2* gradientechoplanar sequence (repetition time/echo time: $2290 / 28 \mathrm{~ms}$; bandwidth: $1680 \mathrm{~Hz}$; field of view: $192 \times 192 \mathrm{~mm}$; matrix: $96 \times 96$; flip angle: $75^{\circ}$ ); 38 interleaved axial slices were obtained (voxel size: $2 \times 2 \times 2 \mathrm{~mm}^{3}$ ) covering the whole brain and were acquired parallel to the anteroposterior commissure plane.

Each sequence lasted for 356 s, resulting in 156 images. Additional "dummy" volumes were acquired at the beginning of each run to allow the magnetization to stabilize to a steady state before the first real volume. A set of high-resolution three-dimensional T1-weighted images was acquired (3D fast gradient echo inversion recovery sequence, inversion time: $900 \mathrm{~ms}$; repetition time/echo time: $2300 / 4.18 \mathrm{~ms}$; bandwidth: $150 \mathrm{~Hz}$; field of view: $256 \times 256 \mathrm{~mm}$; matrix: $256 \times 256$; flip angle: $9^{\circ}$; voxel size $1 \times 1 \times 1 \mathrm{~mm}^{3}$ ) for anatomical localization. 


\subsection{Statistical analyses}

See Supplementary Material 4 for clinical and behavioral data analyses.

\subsection{1. fMRI data preprocessing and first level analysis}

Data were processed using the SPM5 software (http://www.fil.ion.ucl.ac.uk/spm/software/). A standard data pre-processing protocol was followed (slice timing, movement correction, coregistration with the T1-weighted image and normalization into a standardized Montreal Neurological Institute (MNI) coordinate system space using the transformations computed during the segmentation of the T1-weighted image, and finally spatial smoothing with an isotropic kernel of 8-mm full-width at half-maximum).

An individual statistical parametric map was computed for each subject using a general linear model. Each condition was modeled using an event-related approach and convolved with the canonical hemodynamic response function (HRF) to create regressors of interest (self, general and control). A high-pass filter (cut-off of $128 \mathrm{~s}$ ) was applied and motion realignment parameters included as regressors of non-interest. The first level individual "self versus general" $t$-contrast images were obtained for the HRF estimates.

Because we did not observe any significant $(p<0.005)$ interaction between condition, valence and group at the first and second sessions in a second level analysis of variance (ANOVA), we did not define the first level individual valence $t$-contrast images above and we did not take into account emotional valence in second level analyses (Section 2.7.2).

\subsubsection{Second level analyses}

We carried out second level random effects analyses to investigate both short- and long-term effects of AGO treatment on brain activation during self-referential processing. 
Given the subjectivity in social and affective phenomena leading to person-to-person variability, we focused the fMRI analysis with an a priori region of interest (ROI) approach. To avoid a non-independent analysis from the data set, the ROIs were anatomically defined using coordinates from previous neuroimaging studies of self-referential processing and emotion regulation in healthy and depressed individuals. We identified the nine following ROIs: the dorsomedial prefrontal cortex or Brodmann area (BA) 9 (dmPFC9), dmPFC BA10 (rostral part, dmPFC10), ventromedial prefrontal cortex (vmPFC), PCC, vACC, rostral ACC (rACC), dorsal anterior cingulate (dACC), VLPFC (BA 11), and DLPFC (BA 9) (Phillips et al., 2003b; Drevets et al., 2008; Fales et al., 2009; Lemogne et al., 2009; Lemogne et al., 2011; Kanske et al., 2012; Cisler et al., 2013). The coordinates were used as centers of 5-mm radius spheres (Table 2). The size of the ROIs was selected to favour accuracy of location. For the analyses including a between-group factor (see below), mean reaction times were added as covariates of no interest because of the difference of reaction times between the three groups (see Section 3.3.1).

2.7.2.1. Part I: Short-term effects of 1-week administration of AGO on neural correlates of self-referential processing

An ANOVA with a between-group factor $(\mathrm{AGO} / \mathrm{PBO} / \mathrm{HV})$ and a within-group scan time factor (W0/W1) was performed on the "self versus general" contrast images. The mean signal of all the voxel values in each ROI was extracted at W0 and $\mathrm{W} 1$ for each group (AGO, PBO and HV) using MarsBar software (http://marsbar.sourceforge.net).

\subsubsection{Part II: Long-term effects of 6- to 7-week administration of AGO on neural correlates} of self-referential processing

We performed a paired $t$-test comparing the activation in brain regions involved in self- 
referential processing between $\mathrm{W} 0$ and $\mathrm{W} 7$ in MDD patients. The mean signal of all the voxel values in each ROI was extracted at W0 and at W7 using Marsbar software.

\subsubsection{Part III: Associations between pre-treatment brain activation at WO and clinical} remission at $W 24$

To investigate the possible link between the pre-treatment brain activation and clinical improvement after 24 weeks of antidepressant, we used the 17-item HAM-D as a categorical variable, to split the MDD sample into two groups according to a score cut-off of 7 at W24 (remitters $\leq 7$, non-remitters $>7$ ). Subsequently, we performed an ANOVA comparing brain activation during self-referential processing in ROIs at W0 and W7 (within-group scan time factor) between patients in remission at W24 (i.e., remitters) and those not in remission at W24 (i.e., non-remitters) (between-group factor). The mean signal of all the voxel values in each ROI was extracted at W0 and W7 for each patient group (remitters and non-remitters) using MarsBar software.

\section{Results}

\subsection{Demographic data}

Table 1 summarizes demographic and clinical characteristics of the patient and HV groups. No relevant difference was found between the two groups for age or educational level.

\subsection{Clinical effects}

\subsubsection{Short-term effects of $A G O$}

The two MDD groups did not significantly differ regarding demographic and clinical characteristics at W0 (Table 1).

In the two MDD groups, the mean HAM-D score significantly decreased between W0 (AGO: 
mean=24.4, $\mathrm{SD}=3.3$; PBO: mean=25.3, $\mathrm{SD}=2.9)$ and $\mathrm{W} 1(\mathrm{AGO}$ : mean $=21.4, \mathrm{SD}=4.7$; $\mathrm{PBO}$ : mean $=21.3, \mathrm{SD}=5.1$; main effect of time, $F(1,23)=25.5, p<0.001)$, but this decrease was not clinically significant. No patient was a responder (i.e., HAM-D score reduction $\geq 50 \%$ ) at W1. There was not a significant main effect of group $[F(1,46)=0.07, p=0.8]$ or a group $\times$ time interaction $[F(1,46)=0.45, p=0.5)]$.

\subsubsection{Long-term effects of $A G O$}

The mean score of HAM-D significantly decreased from W0 to W7 (mean=25.1, SD=3.1 vs. mean=13.4, $\mathrm{SD}=5.0 ; t(18)=11.8, p<0.001 ; n=19$ patients) and from $\mathrm{W} 7$ to $\mathrm{W} 24$ (mean $=13.2$, $\mathrm{SD}=5.3$ vs. mean $=8.8, \mathrm{SD}=6.3 ; t(15)=2.8, p=0.014 ; n=16$ patients $)$. At $\mathrm{W} 7,10$ patients $(52.6 \%)$ were responders, seven patients were partial responders (reduction between $30 \%$ and $50 \%$ ) and two patients were non-responders (reduction $<30 \%$ ) [supplementary Table 1]. Among the 16 patients included in analyses regarding remission prediction at $\mathrm{W} 24$, there were nine remitters $(56.2 \%)$ and seven non-remitters. At W0, future remitters and nonremitters did not significantly differ in age, duration of illness, number of depressive episodes or severity of illness according to the HAM-D score. The duration of the current episode was longer in future non-remitters (mean $=7.9$ months, $\mathrm{SD}=4.9$ ) as compared with remitters (mean $=3.1$ months, $\mathrm{SD}=2.3 ; U=11, p=0.03$ ). According to the DSM-IV, there were more severe MDD episodes in future non-remitters compared with remitters $\left(\chi^{2}=5.7, p=0.02\right)$

[supplementary Table 2].

See Supplementary Material 5 for additional clinical effects.

\subsection{Treatment results}

\subsubsection{Behavioural results}

\subsubsection{Short-term effects of $A G O$}

Regarding the reaction times (RTs) during the self and general conditions, we observed a 
main effect of condition $[F(1,72)=5.8 ; p=0.02]$, explained by a slower $\mathrm{RT}$ for the 'self' than the 'general' condition. There was a main effect of group $[F(2,72)=8 ; p<0.001]$ with a longer RT in MDD patients (AGO and PBO) than in healthy controls, the AGO patients being slower than the PBO group (supplementary Table 3). There was no main effect or time, nor any interaction. However, when the valence factor was included in the ANOVA, in addition to a main effect of condition $[F(1,142)=11.5 ; p<0.001]$ and of group $[F(2,142)=14.5 ; p<0.001]$, there was also a significant time $\times$ group interaction $[F(2,142)=3.6 ; p=0.031]$ explained by a shorter RT at time W1 only in the AGO patients. Post hoc tests revealed that depressed patients from the AGO group showed a tendency to decrease their RT time for a "yes" response to positive stimuli in the self condition (W0, mean $=2384 \mathrm{~ms}, \mathrm{SD}=944$; W1, mean $=2195 \mathrm{~ms}, \mathrm{SD}=768 ; \mathrm{p}=0.057)$.

\subsubsection{Long-term effects of $A G O$}

For the reaction times (RTs) during the 'self' and 'general' conditions, we observed a main effect of condition $[F(1,122)=8.9 ; p=0.003]$, explained by a slower RT for the 'self' than the 'general' condition. There was a main effect of group $[F(1,122)=23.5 ; p<0.001]$, with a longer $\mathrm{RT}$ in MDD patients than in healthy volunteers. There was also a main effect of time $[F(1,122)=9.5 ; p=0.002]$ and a time $\times$ group interaction $[F(1,122)=5.8 ; p=0.017]$ explained by a shorter RT at W7 observed only in MDD patients. Post hoc tests revealed that patients decreased their RT for "yes" response for positive and negative stimuli in the 'self' condition (respectively, W0: mean=2239 ms, $\mathrm{SD}=882, \mathrm{~W} 7:$ mean=2025 ms, $\mathrm{SD}=639, p=0.022 ; \mathrm{W0}$ : mean=2348 ms, $\mathrm{SD}=845, \mathrm{~W} 7$, mean $=2013 \mathrm{~ms}, \mathrm{SD}=512, p=0.0008)$ and for positive in the 'general' condition (W0, mean=1913 ms, SD=824; W7, mean=1656 ms, SD=523; $p=0.013$ ). See Supplementary Material 6 for the results for the responses (yes or no) given during the 'self' and 'general' conditions. 


\subsection{2. fMRI results}

\subsubsection{Part I: Short-term effects of 1-week administration of AGO on neural correlates of} self-referential processing

No significant time or group main effects were found, but there was a significant group $\times$ time interaction in the right $\operatorname{VLPFC}(F(2,71)=3.4 ; p=0.038, p$-corrected $=0.3)$ [Fig. 1].

The group $\times$ time interaction found in the VLPFC was explained by a specific effect found in AGO patients: there was a significant decrease of VLPFC activation in AGO patients during the first week of treatment (W0 $>\mathrm{W} 1: t(12)=2.7 ; p=0.004)$ compared with $\mathrm{HV}(\mathrm{AGO}>\mathrm{HV} \times$ $\mathrm{W} 0>\mathrm{W} 1, t(71)=2.6, p=0.006)$ that was not observed in PBO patients. There was indeed a trend toward significance when comparing this decrease in AGO patients versus PBO patients $(\mathrm{AGO}>\mathrm{PBO}$ patients $\times \mathrm{W} 0>\mathrm{W} 1, t(71)=1.6, p=0.056)$. At $\mathrm{W} 1$, the VLPFC activation was no longer different in AGO patients versus HV (Fig. 1).

No correlation was found between VLPFC activation and the HAM-D score (results not shown).

\subsubsection{Part II: Long-term effect of 6/7-week administration of AGO on neural correlates of} self-referential processing

After 6-7 weeks of administration of AGO (W7 vs. W0), MDD patients displayed an increased activation in the $\operatorname{vACC}(t[18]=2.7 ; p=0.008, p$-corrected $=0.075)($ Fig. 2$)$ and a tendency to decrease the activation of the DLPFC $(t[18]=1.4, p=0.087, p$-corrected $=0.6)$. 


\subsubsection{Part III: Associations between pre-treatment brain activation at WO and clinical} remission at $W 24$

Comparing remitters $(n=9)$ and non-remitters $(n=7)$ patients, we observed a significant group main effect in the dmPFC BA10 $(F[1,28]=8.4, p=0.007, p$-corrected=0.07), $\mathrm{PCC}(F[1,28]=5.4$, $p=0.028, p$-corrected $=0.24)$, and DLPFC $(F[1,28]=6.4 ; p=0.017, p$-corrected $=0.16)$ explained by a decreased activation in these regions in future remitters compared with future nonremitters (Fig. 3). There was no group $\times$ time interaction, suggesting that activation in these brain regions remained stable between $\mathrm{W} 0$ and $\mathrm{W} 7$.

\section{Discussion}

This study aimed to assess short-term and long-term effects associated with treatment with an antidepressant drug, agomelatine, on the neural bases of self-referential processing in depressed patients as well as the association between pre-treatment brain activation and remission of depression 6 months later. After 1 week of treatment, only MDD patients receiving agomelatine normalized the hyperactivity of the VLPFC during self-referential processing in relation to healthy volunteers' level. After $6 / 7$ weeks of administration of agomelatine, MDD patients displayed an increased vACC activation during self-referential processing. Finally pretreatment activation in the dmPFC10, PCC and DLPFC during selfreferential processing distinguished future remitters from non-remitters 6 months later. Activation in these regions was lower in remitters than in non-remitters and remained stable after $6 / 7$ weeks of treatment in both groups of patients.

The change of VLPFC activation between baseline and W1 was specifically found in the group of depressed patients treated with agomelatine. Two studies investigated the early effects of antidepressants on neural responses in depressed patients (Mayberg et al., 2000; Davidson et al., 2003), but the specific early effect of agomelatine compared with placebo on 
the VLPFC in the present study is a new finding in depressed patients.

Beyond regulating negative emotions (Phillips et al., 2003), the VLPFC plays a key role in the processing of positive emotional stimuli in major depression. Light et al. (2011) recently found that depressed patients exhibiting lesser VLPFC activity during the inhibition of positive affect showed a greater reduction of anhedonia after 8 weeks of antidepressant treatment. Interestingly, there is some evidence that agomelatine may exert early specific effects on anhedonia in major depression (Di Giannantonio et al., 2011; Martinotti et al., 2012). Although we did not include an anhedonia rating scale in the present study, we may hypothesize that the early effects of agomelatine on anhedonia are mediated by changes on VLPFC activity.

The vACC is part of the subgenual ACC receiving the dopamine (DA) projection from the ventral tegmental area, the origin of the mesolimbic DA system (Drevets et al., 1997). Drevets and collaborators hypothesized that the reduced vACC activity in MDD could be associated with diminished stimulation of mesolimbic DA release, resulting in reduced incentive motivation and anhedonia in MDD (Drevets et al., 2008; Cléry-Melin et al., 2011). The modulation of VACC and VLPFC activations associated with agomelatine treatment may relate to its unique pharmacological action. Both the stimulation of melatoninergic receptors and the blockade of 5-HT2c receptors increase dopamine and noradrenaline neurotransmission and their release has been observed in the fronto-cortical dopaminergic and noradrenergic pathways (Millan et al., 2003; Chenu et al., 2013).

PCC, dmPFC10 and DLPFC activation during self-referential processing at baseline clearly differentiated remitters from non-remitters. We selected the remission as the targeted endpoint of our analysis. Remission can be defined as a complete or near complete absence of symptoms, and patients achieving remission have a lower likelihood of depressive recurrence 
and a better psychosocial functioning compared with patients with residual depressive symptoms.

Using this endpoint, we did not replicate the findings of hyperactivity of the rostral region of anterior cingulate cortex (rACC) associated with prognosis. The importance of rACC activity in treatment outcome of depression has been associated with response to several treatments rather than with remission. Brain regions associated with the prediction of treatment response may be different from brain regions associated with the prediction of remission. Studies with larger samples are needed to test this hypothesis and to define the neurobiological heterogeneity of patients with different long-term outcomes.

The pre-treatment activation of dmPFC10 did not change after $6 / 7$ weeks of antidepressant. A persistent hyperactivation of the dmPFC during self-referential processing in MDD patients compared with controls has been previously described following 9 weeks of antidepressant drug treatment (Lemogne et al., 2010). Given the strong relationships between dmPFC and self-referential processing (Lemogne et al., 2012), we may hypothesize that cognitive behavior therapy (CBT), by addressing cognitive biases such as excessive self-focus, would target the dorsal part of the medial prefrontal cortex more specifically than antidepressant drugs. Indeed, while CBT influences both the ventral and dorsal medial frontal regions (Siegle et al., 2012; Yoshimura et al., 2013), a specific modulation by CBT on the dmPFC was described when compared with paroxetine (Goldapple et al., 2004) in the treatment of depression. Further studies comparing drugs and CBT (McGrath et al., 2013) are needed to test if reducing activity in the dmPFC during self-referential processing is a mechanism underlying clinical changes specific to CBT.

This preliminary study is not without limitations. First, although the MDD groups were clinically well characterized and carefully matched with the control group, our samples of 
patients and healthy controls were relatively small. The further subdivision of the MDD patients into remitted and non-remitted groups increases this limitation. In addition, although this study was not a therapeutic trial, the attrition rate was high because of the constraints of and the controls related to possible bias in the fMRI protocol (mainly excessive head movements and positive drug screening). Many group differences in this first study did not survive correction for multiple comparisons, thus emphasizing the need for replication of this study in studies involving larger groups of patients. Therefore, our findings must be interpreted with caution. For ethical reasons, we were not able to introduce a prolonged placebo arm in depressed patients. Moreover, we did not include a group of healthy controls receiving agomelatine. Finally, we do not know whether the predictive value of the dmPFC and the PCC is specific to agomelatine or even to antidepressant drugs in general.

In summary, acute depressed patients receiving the antidepressant agomelatine showed a normalized VLPFC activation during self-referential processing after 1 week of treatment, before significant clinical improvement of depressive symptoms. Whereas 6-7 weeks administration of the antidepressant was associated with a selective increase of vACC activation during self-referential processing, the pre-treatment activation of the dmPFC, PCC and DLPFC was associated with clinical remission at 6 months. 


\section{Acknowledgments}

This study was fully funded by Servier. The authors thank the psychiatrists from GICIPI (Groupe d'investigateurs cliniciens indépendants pour la réalisation d'études pivotales de qualité) for the inclusion and the assessment of the MDD patients, the Clinical Investigation Center of Pitié-Salpêtrière Hospital and the CENIR (Centre de Neuroimagerie de Recherche) for their technical assistance.

\section{Author disclosures}

P. Delaveau was supported by Institut de Recherches Internationales Servier (IRIS). M.

Jabourian and J. Laredo are employees of Servier. P. Fossati received honoraria for this study. P. Fossati and S. Lehéricy received research grants from Servier. C. Lemogne has accepted paid speaking engagements in industry-sponsored symposia from Astra Zeneca, Lundbeck, Pierre Fabre, Pfizer and Servier.

\section{Role of funding source}

Funding of these authors had no further role in the analysis and interpretation of data, in the writing of the report and in the decision to submit the paper for publication. P. Delaveau had full access to all of the data in the study and takes responsibility for the integrity of the data and the accuracy of the data analysis.

\section{Contributors}

P. Delaveau wrote the protocol, acquired data, managed the literature searches, undertook the statistical analysis, interpreted the results, and wrote the first draft of the manuscript. $\mathrm{M}$ Jabourian designed the study, wrote the protocol, managed the literature searches, and critical revision of document. C. Lemogne designed the study, managed the literature searches and 
critical revision of the manuscript. N. Allaïli, W. Choucha, and N. Girault assessed the patients and critically reviewed the manuscript. S. Lehericy and J. Laredo managed the literature searches and critical revision of the manuscript. P. Fossati designed the study, wrote the protocol, coordinated the study, assessed the patients, interpreted the results, managed the literature searches, and advised in the first draft of the manuscript. All authors contributed to and have approved the final manuscript.

\section{References}

Chenu, F., El Mansari, M., Blier, P., 2013. Electrophysiological effects of repeated administration of agomelatine on the dopamine, norepinephrine, and serotonin systems in the rat brain. Neuropsychopharmacology $38,275-284$.

Cisler, J.M., James, G.A., Tripathi, S., Mletzko, T., Heim, C., Hu, X.P., Mayberg, H.S., Nemeroff, C.B., Kilts, C.D., 2013. Differential functional connectivity within an emotion regulation neural network among individuals resilient and susceptible to the depressogenic effects of early life stress. Psychol. Med. 43, 507-518.

Cléry-Melin, M.L., Schmidt, L., Lafargue, G., Baup, N., Fossati, P., Pessiglione, M., 2011. Why don't you try harder? An investigation of effort production in major depression. PLoS One 6, e23178.

Davidson, R., Irwin, W., Anderle, M.J., Kalin, N.H., 2003. The neural substrates of affective processing in depressed patients treated with venlafaxine. Am. J. Psychiatry 160, 64-75.

de Bodinat, C., Guardiola-Lemaitre, B., Mocaër, E., Renard, P., Muñoz, C., Millan, M.J., 2010. Agomelatine, the first melatonergic antidepressant: discovery, characterization and development. Nat. Rev. Drug Discov. 9, 628-642. 
Di Giannantonio, M., Di Iorio, G., Guglielmo, R., De Berardis, D., Conti, C.M., Acciavatti, T., Cornelio, M., Martinotti, G., 2011. Major depressive disorder, anhedonia and agomelatine: an open- label study. J. Biol. Regul. Homeost. Agents 25, 109-114.

Di Simplicio, M., Norbury, R., Harmer, C.J., 2012. Short-term antidepressant administration reduces negative self-referential processing in the medial prefrontal cortex in subjects at risk for depression. Mol. Psychiatry 17, 503-510.

Drevets, W.C., Price, J.L., Simpson, J.R. Jr, Todd, R.D., Reich, T., Vannier, M., Raichle, M.E., 1997. Subgenual prefrontal cortex abnormalities in mood disorders. Nature 386, 824827.

Drevets, W.C., Savitz, J., Trimble, M., 2008. The subgenual anterior cingulate cortex in mood disorders. CNS Spectrums 13, 663-681.

Fales, C.L., Barch, D.M., Rundle, M.M., Mintun, M.A., Mathews, J., Snyder, A.Z., Sheline, Y.I., 2009. Antidepressant treatment normalizes hypoactivity in dorsolateral prefrontal cortex during emotional interference processing in major depression. J. Affect. Disord. 112, 206-211

Geday, J., Gjedde, A., Boldsen, A.S., Kupers, R., 2003. Emotional valence modulates activity in the posterior fusiform gyrus and inferior medial prefrontal cortex in social perception. Neuroimage 18, 675-684.

Goldapple, K., Segal, Z., Garson, C., Lau, M., Bieling, P., Kennedy, S., Mayberg, H., 2004. Modulation of cortical-limbic pathways in major depression: treatment-specific effects of cognitive behavior therapy. Arch. Gen. Psychiatry 61, 34-41.

Guy, W., 1976. Clinical Global Impressions, in: Guy, W. (ed.), ECDEU Assessment Manual for Psychopharmacology, revised. NIMH, US Department of Health, Education, and Welfare, 
Rockville, MD, pp. 218-222.

Hamilton, M., 1960. A rating scale for depression. J. Neurol. Neurosurg. Psychiatry 23, 5662.

Harmer, C.J., Bhagwagar, Z., Perrett, D.I., Völlm, B.A., Cowen, P.J., Goodwin, G.M., 2003. Acute SSRI administration affects the processing of social cues in healthy volunteers. Neuropsychopharmacology 28, 148-152.

Harmer, C.J., Shelley, N., Cowen, P., Goodwin, G., 2004. Increased positive versus negative affective perception and memory in healthy volunteers following selective serotonin and norepinephrine reuptake inhibition. Am. J. Psychiatry 161, 1256-1263.

Harmer, C.J., O'Sullivan, U., Favaron, E., Massey-Chase, R., Ayres, R., Reinecke, A., Goodwin, G.M., Cowen, P.J., 2009. Effect of acute antidepressant administration on negative affective bias in depressed patients. Am. J. Psychiatry 166, 1178-1184.

Kanske, P., Heissler, J., Schönfelder, S., Wessa, M., 2012. Neural correlates of emotion regulation deficits in remitted depression: the influence of regulation strategy, habitual regulation use, and emotional valence. Neuroimage 61, 686-693.

Lang, P.J., Bradley, M.M., Cuthbert, B.N. 2005. International Affective Picture System (IAPS). Digitized photographs, instruction manual and affective ratings. Technical Report A6, NIMH Center for the Study of Emotion and Attention, University of Florida, Gainesville, FL.

Lemogne, C., le Bastard, G., Mayberg, H., Volle, E., Bergouignan, L., Lehéricy, S., Allilaire, JF, Fossati, P., 2009. In search of the depressive self: extended medial prefrontal network during self- referential processing in major depression. Soc. Cogn. Aff. Neurosci. 4, 305-312. 
Lemogne, C., Mayberg, H., Bergouignan, L., Volle, E., Delaveau, P., Lehéricy, S., Allilaire, J.F., Fossati, P., 2010. Self-referential processing and the prefrontal cortex over the course of depression: a pilot study. J. Affect. Disord. 124, 196-201.

Lemogne, C., Gorwood, P., Bergouignan, L., Pélissolo, A., Lehéricy, S., Fossati, P., 2011. Negative affectivity, self-referential processing and the cortical midline structures. Soc. Cogn. Aff. Neurosci. 6, 426-433.

Lemogne, C., Delaveau, P., Freton, M., Guionnet, S., Fossati, P., 2012. Medial prefrontal cortex and the self in major depression. J. Affect. Disord. 136, e1-e11.

Light, S.N., Heller, A.S., Johnstone, T., Kolden, G.G., Peterson, M.J., Kalin, N.H., Davidson, R.J., 2011. Reduced right ventrolateral prefrontal cortex activity while inhibiting positive affect is associated with improvement in hedonic capacity after 8 weeks of antidepressant treatment in major depressive disorder. Biol. Psychiatry 70, 962-968.

Martinotti, G., Sepede, G., Gambi, F., Di Iorio, G., De Berardis, D., Di Nicola, M., Onofrj, M., Janiri, L., Di Giannantonio, M., 2012. Agomelatine versus venlafaxine XR in the treatment of anhedonia in major depressive disorder: a pilot study. J. Clin. Psychopharmacol. $32,487-491$.

Matthews, SC, Simmons, AN, Strigo, IA, Arce, E, Stein, MB, Paulus, MP., 2010. Escitalopram attenuates posterior cingulate activity during self-evaluation in healthy volunteers. Psychiatry Res. 182, 81-87.

Mayberg, H.S., Brannan, S.K., Tekell, J.L., Silva, J.A., Mahurin, R.K., McGinnis, S., Jerabek, P.A., 2000. Regional metabolic effects of fluoxetine in major depression: serial changes and relationship to clinical response. Biol. Psychiatry 48, 830-843. 
McGrath, C.L., Kelley, M.E., Holtzheimer, P.E., Dunlop, B.W., Craighead, W.E., Franco, A.R., Craddock, R.C., Mayberg, H.S., 2013. Toward a neuroimaging treatment selection biomarker for major depressive disorder. JAMA Psychiatry 70, 821-829.

Millan, M.J., Gobert, A., Lejeune, F., Dekeyne, A., Newman-Tancredi, A., Pasteau, V., Rivet, J.M., Cussac, D., 2003. The novel melatonin agonist agomelatine (S20098) is an antagonist at 5- hydroxytryptamine $2 \mathrm{C}$ receptors, blockade of which enhances the activity of frontocortical dopaminergic and adrenergic pathways. J. Pharmacol. Exp. Ther. 306, 954-964.

Mor, N., Winquist, J., 2002. Self-focused attention and negative affect: a meta-analysis. Psychol. Bull. 128, 638-662.

Nejad, A.B., Fossati, P., Lemogne, C., 2013. Self-referential processing, rumination, and cortical midline structures in major depression. Front. Hum. Neurosci. 7, 666.

Phillips, M.L., Drevets, W.C., Rauch, S.L., Lane, R., 2003a. Neurobiology of emotion perception I. The neural basis of normal emotion perception. Biol. Psychiatry 54, 504-514.

Phillips, M.L., Drevets, W.C., Rauch, S.L., Lane, R., 2003b. Neurobiology of emotion perception II: Implications for major psychiatric disorders. Biol. Psychiatry. 54,515-528.

Sheehan, D.V., Lecrubier, Y., Sheehan, K.H., Amorim, P., Janavs, J., Weiller, E., 1998. The Mini-International Neuropsychiatric Interview (M.I.N.I.): the development and validation of a structured diagnostic psychiatric interview for DSM-IV and ICD-10. J. Clin. Psychiatry 59, $22-33$

Siegle, G.J., Thompson, W.K., Collier, A., Berman, S.R., Feldmiller, J., Thase, M.E., Friedman, E.S., 2012. Toward clinically useful neuroimaging in depression treatment: prognostic utility of subgenual cingulate activity for determining depression outcome in 
cognitive therapy across studies, scanners, and patient characteristics. Arch. Gen. Psychiatry 69, 913-924.

Taylor, D., Sparshatt, A., Varma, S., Olofinjana, O., 2014. Antidepressant efficacy of agomelatine: meta-analysis of published and unpublished studies. BMJ. 348, g1888.

Yoshimura, S., Okamoto, Y., Onoda, K., Matsunaga, M., Ueda, K., Suzuki, S., 2010. Rostral anterior cingulate cortex activity mediates the relationship between the depressive symptoms and the medial prefrontal cortex activity. J. Affect. Disord. 122, 76-85.

Yoshimura, S, Okamoto, Y, Onoda, K, Matsunaga, M, Okada, G, Kunisato, Y, Yoshino, A, Ueda, K, Suzuki, SI, Yamawaki, S., 2013. Cognitive behavioral therapy for depression changes medial prefrontal and ventral anterior cingulate cortex activity associated with selfreferential processing. Soc. Cogn. Affect. Neurosci. 9, 487-493. 


\section{Figure legends}

Fig. 1. Mean BOLD signal from the VLPFC, in MDD patients treated by placebo (PBO) or agomelatine (AGO) and healthy volunteers (HV), at W0 and W1 (MNI coordinates, 3044 11). The bars graphs plot the contrast estimates (arbitrary units) between self-referential and general conditions $( \pm 90 \%$ confidence interval) per group and scan time.

Abbreviations: VLPFC: ventrolateral prefrontal cortex.

Fig. 2. Mean BOLD signal from the vACC (MNI coordinates, 532 -2) per scan times (W0 and W7) in MDD patients ( $n=19)$. The bar graphs plot the contrast estimates (arbitrary units) between self -referential and general conditions ( \pm standard error for mean). Abbreviations: vACC : ventral anterior cingulate cortex.

Fig. 3. A. Mean BOLD signal from the regions of interest in future remitters and nonremitters, at W0 and W7: A. dmPFC10 (MNI coordinates, 160 6), B. PCC (-3 -54 18), C. DLPFC (36 26 33). The bars graphs plot the contrast estimates (arbitrary units) between selfreferential and general conditions ( $\pm 90 \%$ confidence interval) per group and scan time (the points represent the individual values of each patient).

Abbreviations: dmPFC10: dorsomedial prefrontal cortex Brodmann area 10, PCC: posterior cingulate cortex, DLPFC: dorsolateral prefrontal cortex. 
Table 1. Demographic and clinical characteristics of major depressive disorder patients and healthy volunteers (part I) at baseline

\begin{tabular}{|c|c|c|c|}
\hline Characteristics & $\begin{array}{l}\text { AGO patients } \\
\text { group } \\
(n=13)\end{array}$ & $\begin{array}{l}\text { PBO patients } \\
\text { group } \\
(n=12)\end{array}$ & $\begin{array}{l}\text { HV group } \\
\qquad(n=14)\end{array}$ \\
\hline $\begin{array}{l}\text { Age (years, mean } \pm \text { SD) } \\
\min -\max \end{array}$ & $\begin{array}{cc}41.8 & ( \pm 8.0) \\
27-52 & \end{array}$ & $\begin{array}{cc}40.1 & ( \pm 7.6) \\
27-50 & \end{array}$ & $\begin{array}{cc}41.6 \quad( \pm 7.4) \\
26-53\end{array}$ \\
\hline Right handed/left handed (n/n) & $13 / 0$ & $11 / 1$ & $13 / 1$ \\
\hline Education (years, mean \pm SD) & $14.4 \quad( \pm 1.7)$ & $( \pm 2.3)$ & $13.0 \quad( \pm 2.3)$ \\
\hline $\begin{array}{l}\text { DSM-IV classification: } \\
\text { - Recurrent episode }(n) \\
\text { - Severity } \quad \text { Moderate }(n) \\
\text { Severe without psychotic feature }(n) \\
\text { - Melancholic features }(n)\end{array}$ & $\begin{array}{l}13 \\
5\end{array}$ & $\begin{array}{l}5 \\
7 \\
8\end{array}$ & $\begin{array}{l}- \\
- \\
- \\
-\end{array}$ \\
\hline Duration of the disease (years, mean \pm SD) & $16.0 \quad( \pm 9.7)^{\mathrm{a}}$ & $10.0 \quad( \pm 7.2)^{\mathrm{a}}$ & - \\
\hline Current episode duration (months, mean \pm SD) & $5.4 \quad( \pm 4.2)$ & $3.4 \quad( \pm 2.9)$ & - \\
\hline $\begin{array}{l}\text { Number of previous depressive episodes (including } \\
\text { the current one) }(\text { mean } \pm \text { SD) }\end{array}$ & $( \pm 1.2)$ & $2.4 \quad( \pm 1.2)$ & - \\
\hline HAMD-D-17 score $($ mean \pm SD) & $24.4 \quad( \pm 3.3)$ & $25.3 \quad( \pm 2.9)$ & - \\
\hline CGI severity of illness score (mean \pm SD) & $( \pm 0.6)$ & $( \pm 0.5)$ & - \\
\hline
\end{tabular}

$\mathrm{AGO}=$ agomelatine treatment; $\mathrm{PBO}=$ placebo treatment; $\mathrm{HV}=$ healthy volunteers; $\mathrm{SD}=$ standard deviation; $n=$ number of subjects.

${ }^{\mathrm{a}} p$ for difference between $\mathrm{AGO}$ and $\mathrm{PBO}$ patients $=0.09$. 
Table 2. Name (abbreviations) and Montreal Neurological Institute coordinates of the regions of interest comprising self-referential and emotion regulation processes networks

\begin{tabular}{|l|l|l|l|}
\hline Name & $x$ & $y$ & $z$ \\
\hline Dorsomedial prefrontal cortex, BA 9 (dmPFC9) & -6 & 26 & 40 \\
\hline Dorsomedial prefrontal cortex, BA 10 (dmPFC10) & 1 & 60 & 6 \\
\hline Ventromedial prefrontal cortex (vmPFC) & -6 & 42 & -12 \\
\hline Posterior cingulate cortex (PCC) & -3 & -54 & 18 \\
\hline Dorsal anterior cingulate cortex (dACC) & 1 & 14 & 30 \\
\hline Rostral anterior cingulate cortex (rACC) & 2 & 36 & 6 \\
\hline Ventral Anterior cingulate cortex (vACC) & 5 & 32 & -2 \\
\hline Dorsolateral prefrontal cortex (DLPFC) & \pm 36 & 26 & 33 \\
\hline Ventrolateral prefrontal cortex (VLPFC) & \pm 30 & 44 & 11 \\
\hline
\end{tabular}

$\mathrm{BA}=$ Brodmann area 


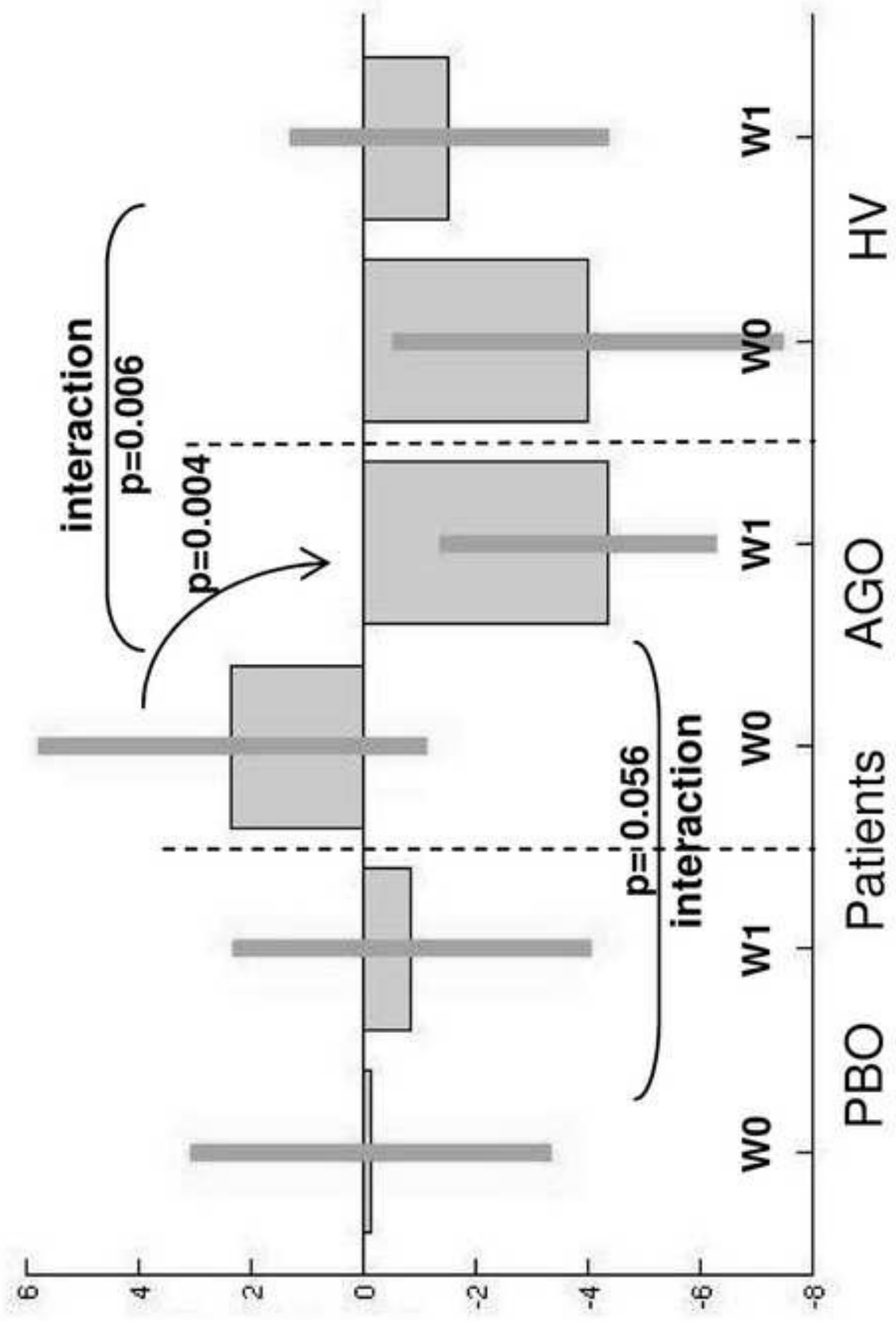



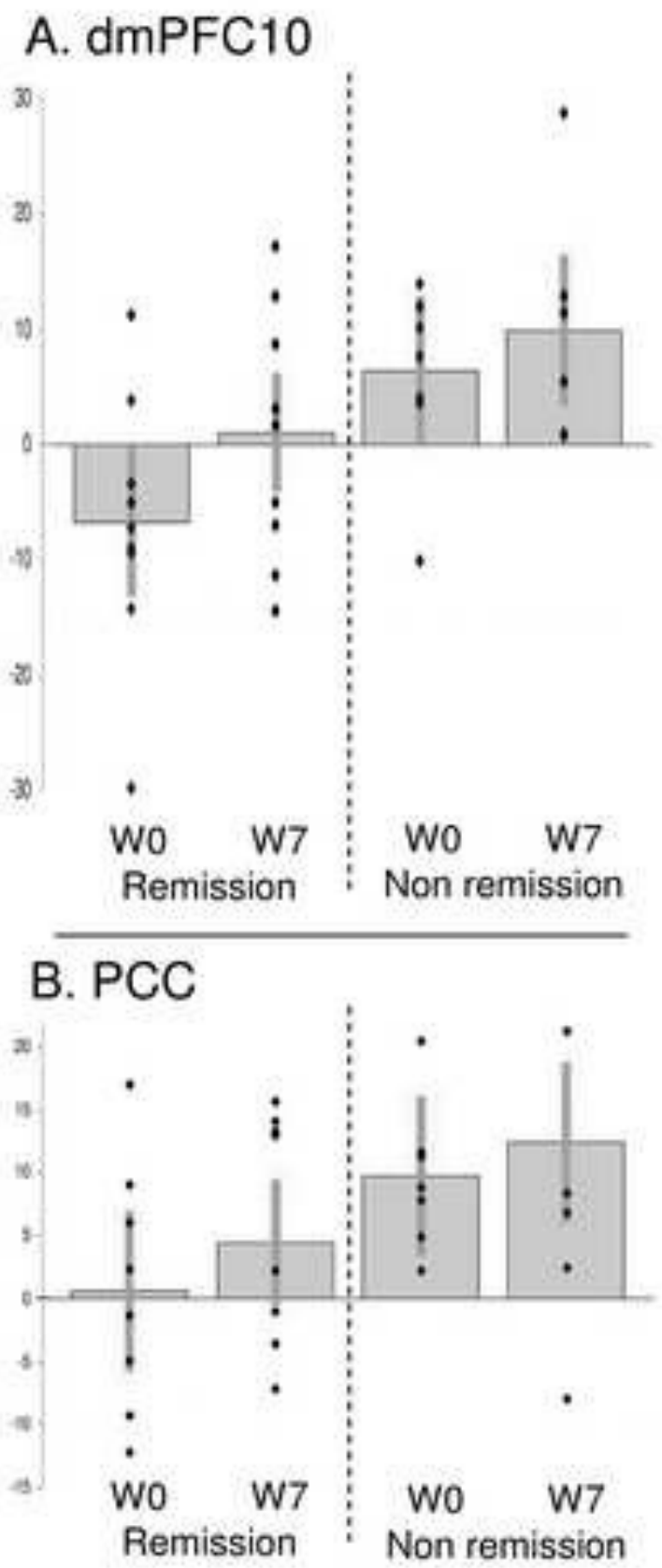

C. DLPFC

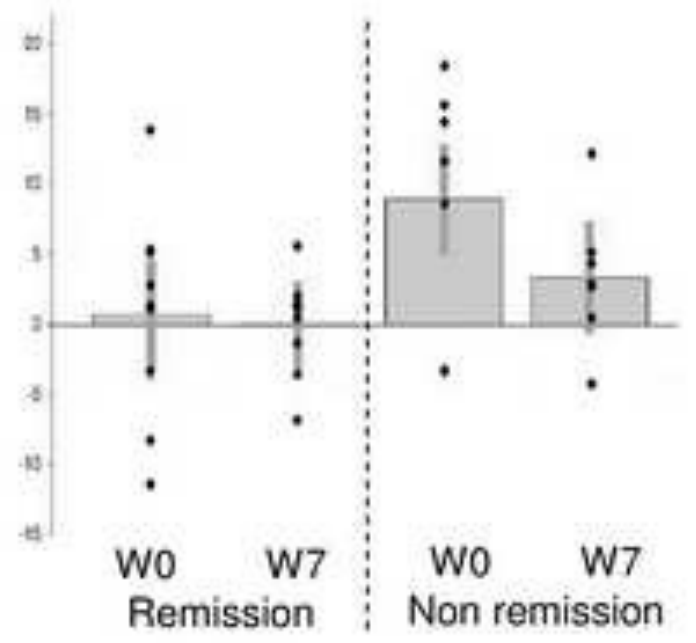




\section{Highlights}

- Short-term and long-term brain effects of a new antidepressant compound were investigated in major depression without anxiety disorder

- Depressed patients were scanned during a self-referential task on three occasions, before beginning the treatment, after 1 week and after 7 weeks of treatment.

- The antidepressant normalized VLPFC activation after 1 week of treatment and increased the ventral ACC activation after six/seven weeks of treatment

- The pre-treatment activation of dorsomedial PFC, and precuneus was associated with clinical remission at 6 months.

- This new antidepressant had short- and long-term effects on brain structures involved in anhedonia and emotional regulation during self-referential processing 